Research Article

\title{
Motion Sensor Monitoring and Ability Evaluation of Sprint Hurdle Swing Training under the Background of Wireless Communication Network
}

\author{
Changliang Huang $\mathbb{D}^{1}$ and Yuting $\mathrm{Xu}^{2}$ \\ ${ }^{1}$ School of Competitive Sports and Physical Education, Shandong Sport University, Rizhao 276800, Shandong, China \\ ${ }^{2}$ School of Physical Education Department, Harbin Institute of Technology (Weihai), Weihai 264209, Shandong, China \\ Correspondence should be addressed to Changliang Huang; huangchangliang@sdpei.edu.cn
}

Received 11 August 2021; Revised 15 September 2021; Accepted 23 September 2021; Published 1 October 2021

Academic Editor: Sang-Bing Tsai

Copyright (c) 2021 Changliang Huang and Yuting Xu. This is an open access article distributed under the Creative Commons Attribution License, which permits unrestricted use, distribution, and reproduction in any medium, provided the original work is properly cited.

\begin{abstract}
The struggle with obstacles has very high technical requirements, and technical movements are particularly complex, requiring the coordination of the entire body of athletes. This article aims to study the training methods and training plans of sprinters, trying to gradually improve the waist strength, abdomen, and hip muscles of sprinters. Through basic strength training, we improve the ability of the body to coordinate strength and give full play to the core strength and sprint technique of the athletes. This paper proposes a motion sensor detection technology, which provides an effective tool for testing and analysis of sports training. The research object of this article is the hurdle running special physical speed, quality, and training methods. Based on a large amount of literature and theoretical analysis, an experimental group and a control group are set up, and an inductive analysis is carried out. The four aspects of peak power and half squat 1RM are discussed and analyzed in detail. The experimental results in this paper show that the extensor muscles of the experimental group increased by $56.5 \mathrm{~J}$ and $51.55 \mathrm{~J}$, respectively, and the increase rates were $24.72 \%$ and $19.66 \%$, respectively. In the control group, the extensor muscles increased by $85 \mathrm{~J}$ and $52 \mathrm{~J}$ under the test conditions of $60 \%$ and $300 \%$, respectively, with an increase rate of $38.2 \%$ and $51.2 \%$, respectively; the flexors increased by $32.6 \mathrm{~J}$ and $22 \mathrm{~J}$, respectively, with an increase rate of $36.25 \%$ and $37.28 \%$, respectively.
\end{abstract}

\section{Introduction}

1.1. Background. The pace of technological development is accelerating, and mankind has entered the information age. As the most important and basic information acquisition technology, sensor technology has also been developed by leaps and bounds. Sensor information technology gradually develops from simplification to integration. Since the end of the last century, fieldbus technology has been applied to sensor networks. People use it to create smart sensor networks. A large number of multifunctional sensors are used and connected through wireless technology, and wireless sensor networks are gradually taking shape. As the wireless sensor network enters people's lives, people put forward higher requirements for the wireless sensor network, which promotes the dynamic development of the wireless sensor network [1]. Simply put, the wireless dynamic sensor network is to add dynamic nodes on the basis of the wireless sensor network. The dynamic node is based on the original node of the wireless network sensor, adding a motion control part, which will cause the initial static node to move and add many new functions. The wireless dynamic sensor network is the most advanced technology in the wireless sensor network.

1.2. Significance. On the one hand, sensor network technology is a new type of network technology that incorporates radio communication technology. Sensor technology, MEMS technology, and distributed information processing technology are intercepting radio sensor networks. The information of the objects or environment in the monitoring 
area are collected and processed through node cooperation; the processed information are transmitted to the end-users of the target network. Wireless sensor networks are widely used, not only in the fields of environmental science and military but also in the field of daily medical care, smart homes, and other commercial areas. On the other hand, there is the meaning of sprint hurdles. From Liu Xiang's gold medal in the hurdle race at the Athens Olympics to the young player Xie Wenjun in the last 10 meters of the Shanghai Athletics Federation Diamond League, he overtakes his opponent and stands on the podium. China is in the sprint event. Sports performance is constantly improving. It can be found that the improvement of sports performance is not only limited to good technical aspects but also has a leap in special physical training. The reason Chinese hurdles have a place in international track and field must have their own unique training methods and methods. Even so, we should continue to improve and summarize the future development of the project and in-depth analysis of the shortcomings of unilateral training. Dyskinesia is a phenomenon in which voluntary movement is excited, inhibited, or cannot be controlled by the will. It is common in mental disorders, neurological diseases, and trauma.

1.3. Related Work. The development of motion sensor detection technology provides an effective tool for testing and analysis of sports training. Pinto et al. proposes a new technical solution for marker-based human motion capture, called WirelessSyncroVision (WSV). The proposed solution is to synchronize the image acquisition in the remote multicamera with the opening time of the active marker and adopt a robust method called adaptive and robust synchronization (ARS), and finally, it can be extended to other than $\mathrm{PbD}$ equipment, such as motion capture, motion analysis, and remote sensing systems [2]. Holmstrup et al. examined the effect of an 8 week KBS intervention on sprinting for leisurely active women, compared with an 8 week intervention using a stiff leg deadlift (SDL). After pretests measuring 30-meter sprint and reverse vertical jump performance, participants were divided into KBS $(n=8)$ and SDL $(n=10)$ groups according to the sprint time. After getting familiar with the exercises, KBS met twice a week, using Tabata intervals $(20 \mathrm{~s}$ of work, $10 \mathrm{~s}$ of rest, and 8 rounds) for swings, emphasizing fast and explosive rhythms. It is concluded that future research should seek to determine the appropriate number and strength of KBS components for sprint programming [3]. Dobbin et al. determined the utility of running-only and rugby-specific intraseasonal sprint interval interventions among professional rugby league players. The research method is to assign 31 professional college rugby players to rugby-specific (SITr/s, $n=16$ ) or running-only (SITr, $n=15)$ sprint interval training groups. The measurement of speed, strength, ability to change direction, prone yo-yo intermittent recovery test (yoyo IR1) performance, and heart rate recovery before and after the 2 week intervention, as well as the submaximal response to prone yo-yo IR1, indicates that specific exercises should be considered. The moves are incorporated into the season training of Rugby Union players [4]. Gil and Lee proposed to reduce the athlete's personal recording time by applying the seven-step method. The authors conducted a kinematic analysis of the seven-step method and the generally accepted eight-step method of the current Korean hurdle record holder $(13.43 \mathrm{~s})$ and reached a conclusion about the seven-step method [5]. Misaki et al. proposed to check the acceleration and deceleration curves of the ground reaction force from the entire hurdle sprint to the fifth hurdle. By analyzing the performance of four male college hurdlers, the force applied between the four steps in the interval of the hurdle sprint was obtained. The curves are different, indicating that the role of each step in an interval is different [6]. Camacho-Cardenosa et al. analyzed the impact of the new low-dose repetitive sprint training on short- and long-term physical performance of team sports athletes in hypoxia (RSH). By testing before and after 4 weeks of supervised specific training and 2 weeks after stopping training, it is concluded that lower dose repetitive sprint training under hypoxic conditions can increase the maximum power and sprint of the hypoxic group of team sports athletes times [7]. Although the analysis is well done, some theories do not have practical significance. There are some problems with the viewpoints and methods used by these scholars in the research process.

1.4. Innovation. The innovations of this article are as follows. (1) The first is the innovation of the topic selection angle. This article is a new perspective from the perspective of topic selection. At present, there are not many researches that integrate wireless communication, sprint hurdles, motion sensors, physical fitness, and training methods. It is of exploratory significance. (2) The second is the innovation of research methods. This paper proposes a sensor optimal placement algorithm, wireless sensor network positioning algorithm, and amorphous algorithm. These algorithms are used to study the monitoring and performance of motion sensors. In addition, setting up an experimental group and a control group for experiments has high theoretical value and exploratory significance. (3) The third is the innovation of project practice. The conclusions drawn can provide better services for track and field training practice, to provide certain theoretical guidance and practical application value for the athletic fitness of track and field sprinters.

\section{Related Research Technology}

2.1. Sensor Optimal Placement Algorithm. With the rapid development of precision manufacturing technology, computer technology, and information processing technology, increasingly sensors have been applied. When the information collected from each sensor is processed and isolated separately, not only the connection between the sensor information is interrupted but also the attributes of the relevant environment that may be contained in the organic combination of information are also lost, which leads to a waste of information resources and this leads to a high level of information processing. Information fusion 
technology can effectively solve the above problems. It uses signal processing, artificial intelligence, mathematical statistics, control principles and biology, and other related theories to organically combine the local and incomplete sensory measurements of multiple sensors. These sensors are distributed in different positions and different states and use complementary information to reduce data uncertainty and improve the accuracy and speed of multisensor systems [8].

The workload of calculating the optimal position of the measuring point is often relatively large, and most scholars use the calculation software MATLAB programming [9] to achieve. One of the most commonly used methods is the effective independence method, which calculates effective independent vectors based on all possible measurement points, sorts them according to the independence of the target modal matrix, and iteratively deletes the degrees of freedom.

Assuming that the number of all possible sensor positions is $t$ and the number of sensors to be arranged is $n$, then the response expression of the structure is

$$
a_{n}=\varphi_{n} q+w
$$

where $a$ is the structural modal matrix.

$$
\varphi_{n}=\left(\varphi_{1}, \varphi_{2}, \ldots, \varphi_{n}\right)=\left(\varphi_{i j}\right)_{n * t},
$$

where $\varphi_{1}=\left(\varphi_{1 I}, \varphi_{2 I}, \ldots, \varphi_{s I}\right)^{T}$ represents the $I$ modal vector; $p=\left(p_{1} p_{2} \ldots p_{t}\right)$ is the generalized coordinate; $Q$ is Gaussian white noise with a variance of $\eta^{2} 5$.

The least-squares estimation expression of modal coordinates is as follows:

$$
\widehat{p}=\left[\varphi^{T}{ }_{n} \varphi_{n}\right]^{-1} \varphi^{T}{ }_{n} u_{s} .
$$

The covariance expression of the estimated error of $p=$ $\left(p_{1} p_{2} \ldots p_{t}\right)$ is as follows:

$$
U=L\left[(\widehat{p}-p)(\widehat{p}-p)^{T}\right]=\partial^{2}\left(\left[\varphi_{n}^{T} \varphi_{n}\right]^{-1}\right) .
$$

Let $P=\varphi^{T}{ }_{n} \varphi_{n}, P$ call the Fisher information matrix. It can be seen from the Fisher information matrix that the smaller the value of the covariance of the estimation error, the larger the value of the Fisher information matrix $P$, that is, when the matrix $P$ norm takes the maximum value, the estimate of $p$ is the best estimate. The matrix $P$ can be expressed as follows:

$$
P=\sum_{i=1}^{n}\left[\varphi_{n}^{i}\right]^{T}\left[\varphi_{n}^{i}\right]=\sum_{i=1}^{s} P^{i} .
$$

In the decision-level fusion process, the sensors need to be transformed to obtain independent identity estimation, and then the attributes of each sensor are classified and fused. In the process of data fusion [10], the original data sent by sensors of the same size are directly merged, and then the merged data is analyzed and the feature extracted, and finally, the identity is determined according to the extracted features. In the feature-level fusion process, features are extracted by analyzing the output information of each sensor so that the feature quantity of each observation target is obtained and fused, and finally, the fusion information is used as the evaluation criterion. The combination of decision-making layers provides the best decision-making and recognition performance but requires higher registration accuracy and sensor transmission bandwidth. The fusion of the data layer retains as much original information as possible, but it also leads to greater information processing and poor real-time performance. The merging of the feature layer is relatively simple, compatible with the advantages and disadvantages of the decision layer [11] and the data layer [12] and provides greater flexibility.

2.2. Wireless Sensor Network Positioning Algorithm. Mobile WSNs node positioning algorithms are mainly divided into Monte-Carlo-based positioning algorithms, DVHop positioning algorithms [13], and other positioning algorithms. The Monte Carlo method, also known as the statistical simulation method, refers to the use of random numbers (or more commonly pseudorandom numbers) to solve many computational problems. The Monte Carlo method is an essential type of numerical calculation method guided by the theory of probability and statistics, which was proposed in the mid-1940s due to the development of science and technology and the invention of electronic computers. On this basis, people have proposed many improved positioning algorithms such as MCB, MSL, WMCL, and so on. WSN data collection is shown in Figure 1.

The DV-Hop positioning algorithm [14] is mainly divided into three stages as follows. (1) The first stage obtains the minimum number of hops. When the vector distance exchange law is used to obtain the replacement condition of the anchor node information, the corresponding hop number information is obtained. After that, the broadcast Internet and other media are used for information output, such as the identification of the anchor node [15]. (2) The average hop distance between nodes is obtained in the second stage. The following equation can be uses to calculate the final result:

$$
\text { HopSize }_{i}=\frac{\sum_{i \neq j} \sqrt{\left(h_{i}-h_{j}\right)^{2}+\left(k_{i}-k_{j}\right)}}{\sum_{i \neq j} v_{i j}},
$$

where $v_{i j}$ refers to the minimum number of hops between anchor nodes $i\left(h_{i} k_{j}\right)$ and $j\left(h_{i} k_{j}\right) 3$. After calculating the average hop-per-hop distance of the anchor node, the data is corrected through broadcast information, and other values are discarded after the unknown node has known the first correction value.

$$
a_{i}=\operatorname{HopSize}_{i} \times k_{i j}
$$

(3) Coordinate position calculation: The minimum number and average jump distance measured before the calculation; the average distance and the unknown node to the anchor node are calculated; then obtain the number of multiple nodes to obtain the least square node coordinates [16]. 


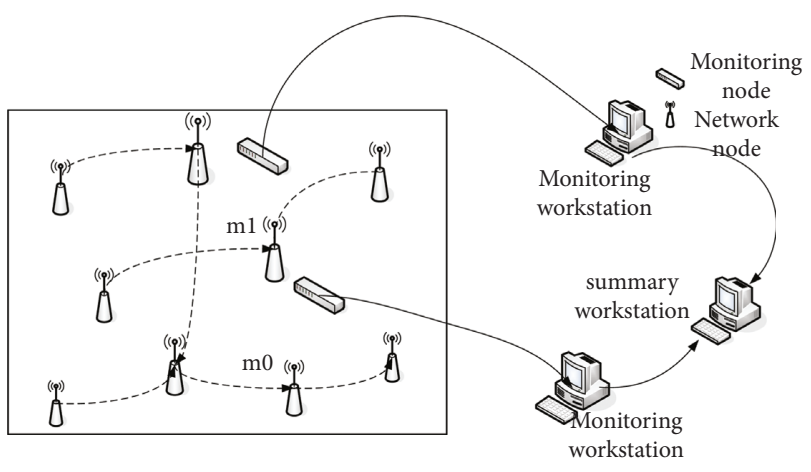

FIGURE 1: WSN data collection.

$$
\begin{aligned}
& \text { HopSize }_{x}=\frac{D 1+D 3}{3+7}, \\
& \text { HopSize }_{y}=\frac{D 1+D 2}{3+9}, \\
& \text { HopSize }_{z}=\frac{D 2+D 3}{7+8} .
\end{aligned}
$$

Using the principle of DV-HOP operation and positioning, three average hop distances are obtained, and the three terms are the distances from the unknown node $\mathrm{O}$ to $x$, $y$, and $z$, and then the least-squares method is used above to obtain the specific unknown node $\mathrm{O}$ coordinate.

The MCL algorithm is a self-localization method for probability estimation based on Bayesian filtering. Its core idea is to use a sample set $B_{t}=\left\{\left(c_{t}^{i}, f_{t}^{i}\right) \mid i=1,2, \ldots, W\right\}$ containing $W$ samples to estimate the posterior probability [17]. Distribution $B_{t}$ is the sample set at time $t, c_{t}^{i}$ is the first $i$ samples, $f_{t}^{i}$ is the weight parameter, and the algorithm has four steps in total. In prediction stage, at time $t-1$, the node position is clear, and then the position distribution of the node at time $t$ has the following formula:

$$
k\left(c_{t} \mid c_{t-1}\right)=\left\{\begin{array}{l}
1 /\left(\pi v_{\max }^{2}\right), d\left(c_{t-1}, c_{t}\right)<v_{\max } \\
0, d\left(c_{t-1}, c_{t}\right) \geq v_{\max }
\end{array}\right.
$$

(i) Filtering stage: In the filtering stage of the algorithm [18], the main work is to filter out the sample points that do not meet the filtering conditions. If $W$ is a one-hop anchor node set, $D$ represents a two-hop anchor node set, $x$ is the communication radius of the anchor node, $z$ is the sample particle, and $y$ is the anchor node.

filter $(l)=(\forall y \in Y, d(l, y) \leq x) \cap(\forall y \in D, x<d(l, y) \leq 2 x)$.

(ii) Re-sampling: When the last stage is over, the number of remaining sample points may be less than $\mathrm{W}$, so the prediction process and filtering stage must be re-executed. When the sample set reaches the standard or the number of samplings is reached, this stage is stopped. The filter fusion block diagram is shown in Figure 2:

(iii) Positioning stage (coordinate calculation): The position coordinates of the unknown node at the moment are calculated by the following formula:

$$
\begin{aligned}
& h_{t}=\sum_{i=1}^{W} \frac{h_{t}^{i}}{W}, \\
& k_{t}=\sum_{i=1}^{W} \frac{k_{t}^{i}}{W} .
\end{aligned}
$$

The schematic diagram of the Monte Carlo algorithm is shown in Figure 3:

\subsection{Amorphous Algorithm}

2.3.1. Solve the Minimum Number of Hops. All anchor nodes broadcast their location information to the entire network so that all nodes can receive this information, and the minimum integer hops from each anchor node [19]; the minimum number of hops from node i to $\mathrm{m}$ can be calculated by the following formula:

$$
Q_{(i, m)}=\frac{\sum_{j \in n b r s(i)} t(j, t)}{|n b r s(i)|+1}-0.8 .
$$

All anchor nodes broadcast their own location information to the whole network so that all nodes can receive this information and get the minimum integer jump [20] from each anchor node, $m, i$ and the number of nodes from $m$ to $i$ can be calculated by using formula (14).

2.3.2. Solve the Distance between Nodes. Equation (2) can be used to calculate the average distance of each hop as follows:

HopSize $=f\left(1+c^{-n}-\int_{-2}^{2} \frac{n}{s \pi}\left(\operatorname{arcos} t-t \sqrt{1-t^{2}}\right) s a\right)$.

Then the distance $p$ between the unknown node and the anchor node is calculated using the minimum number of hops calculated by the average distance of each hop that has been calculated above as follows:

$$
p=\operatorname{HopSize}_{i} \times C_{(i, j)} .
$$

As long as the unknown node [21] finds the estimated distance to the three anchor nodes, the following equations can be listed to calculate the node coordinates:

$$
\left\{\begin{array}{l}
\left(h_{1}-h\right)^{2}+\left(j_{1}-j\right)^{2}=p_{1}^{2} \\
\vdots \\
\left(h_{n}-h\right)^{2}+\left(j_{n}-j\right)^{2}=p_{n}^{2}
\end{array}\right.
$$




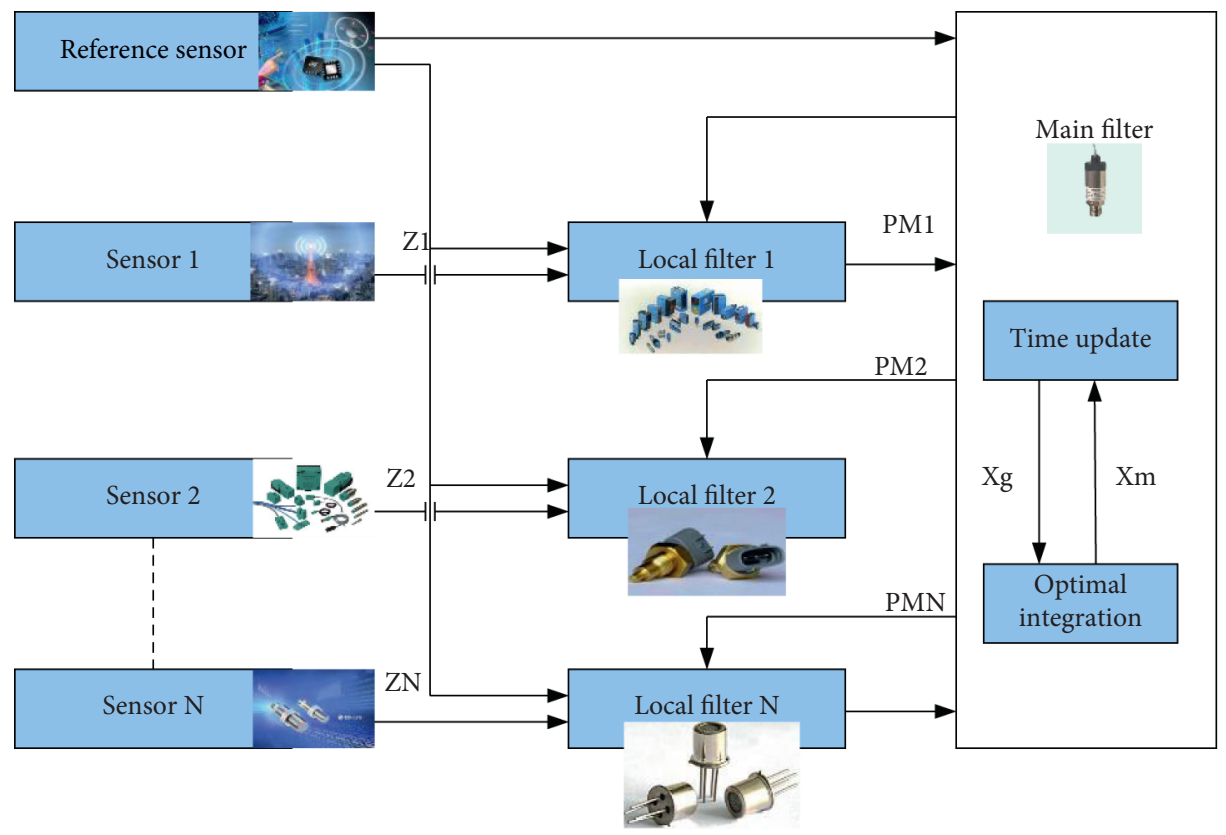

Figure 2: Block diagram of filter fusion.



FIgURE 3: Schematic diagram of Monte Carlo algorithm.

where $\left(h_{1}, j_{1}\right),\left(h_{2}, j_{2}\right),\left(h_{3}, j_{3}\right), \ldots,\left(h_{n}, j_{n}\right)$ is the coordinates of anchor nodes, $(h, j)$ is the coordinates of the unknown node, and the distance between the unknown node and the anchor node is $p_{1}, p_{2}, p_{3}, \ldots, p_{n}$, respectively.

Then the above equations are solved according to the principle of the least-squares method, and then the specific coordinates of the unknown nodes can be obtained as follows:

$$
\begin{aligned}
Q & =\left(D^{T} D\right)^{-1} D^{T} n, \\
W & =\left(\begin{array}{c}
4\left(h_{1}-h_{n}\right) 4\left(j_{1}-j_{n}\right) \\
\vdots \\
4\left(h_{n-1}-h_{n}\right) 4\left(j_{n-1}-j_{n}\right)
\end{array},\right.
\end{aligned}
$$

$$
s=\left\{\begin{array}{l}
h_{1}^{2}-h_{n}^{2}+j_{1}^{2}-j_{n}^{2}+p_{n}^{2}-p_{1}^{2} \\
\vdots \\
h_{n-1}^{2}-h_{n}^{2}+j_{n-1}^{2}-j_{n}^{2}+p_{n}^{2}-p_{n-1}^{2}
\end{array}\right.
$$

Finally, we use equations (17)-(20) to find the coordinates of the unknown node.

\section{Experimental Design of Sprint Hurdle Swing Training Based on Wireless Sensors}

3.1. Subjects. Eighteen men were selected as subjects of track and field sprint events. The average training level of all subjects reached the national athlete level 2. They are in good physical condition, have no injuries such as broken bones or muscle tears, and have no experience in vibration strength training. The 18 subjects were randomly divided into a test group (9) and a control group (9). The test group received vibration training, and the control group received conventional strength training.

To test whether there is a difference between the test indicators of the test group and the control group before the test, the physical condition of the subjects and the four quality indicators are calculated as the quality indicators of $50 \mathrm{~m}$ sprint performance, knee flexion height, anaerobic peak performance, and half squat 1RM. The test and analysis results are shown in Tables 1 and 2:

Table 1 shows the statistical results of the physical function indicators of the test group and the control group. The $P$-value of 0.365 of the age statistics of the test group and the control group indicates that the age of the test group and the control group is different. There was no significant difference between the test group and the control group. The experimental results show that $P$ is 0.896 , indicating that 
there is no significant difference between the two. The $P$-value of the weight statistics result is 0.875 , which indicates that there is no significant difference in weight between the experimental group and the control group.

From Table 2, we can see the statistical results of the four physical fitness indicators of the experimental and the control groups: the $P$-value of the 30 -meter sprint performance of the experimental and the control groups is 0.523 . It shows that the test and the control groups have no significant difference in $30 \mathrm{~m}$ sprayable [22] energy; the statistical result of the knee joint level of the test and the control groups is $0.309(P>0.05)$, indicating that the knee joint level of the test and the control groups has no jump height significant difference; the $P$-value of the anaerobic peak performance statistics of the experimental group and the control group is 0.268 , indicating that there is no significant difference in the anaerobic peak performance of the experimental and the control groups. It includes that there is no significant difference between the experimental and the control groups. Tables 1 and 2 can be concluded that there is no significant difference in physical fitness between the test and the control groups, indicating that the test and the control groups meet the test conditions.

\subsection{Training Program}

3.2.1. Training Program of the Experimental Group. According to the principle of step-by-step training, when athletes learn new skills and movements, they must follow the scientific logic system and the law of development of athletes' understanding, from shallow to deep, from easy to difficult, and from simple to complex. The core strength training studied in this article is an unfamiliar training method for experimental subjects. To help athletes master the training method within a limited time, according to the principle of gradual training, the core strength training of this research is divided into three training stages: core strength training basics phase, core strength training consolidation phase, and core strength training improvement phase. The basic period is 2 to 3 weeks, and the training intensity is relatively small. The purpose is to improve the strength of the waist and abdomen small muscle groups and promote the ability of the nerve-muscle system. The consolidation period is 4 to 5 weeks. The training intensity is moderate; the purpose is to further improve the strength quality of the small waist and abdomen muscles [23], strengthen the coordination ability of the nerve-muscle system, and develop the body balance and stability ability. The improvement phase is 5 to 6 weeks, and the training intensity is high. The purpose is to develop the ability of the nervous system to control balance under unstable conditions and to improve the strength of the small muscle groups of the waist and abdomen.

3.2.2. Training Program of the Control Group. While the experimental group was performing core strength training, the control group used traditional strength training methods for waist and abdominal muscle training. The main equipment was sponge pads and barbells. The training
TABle 1: Pre-experiment information statistics of subjects in the experimental group and control group.

\begin{tabular}{lccc}
\hline Group $(n=9)$ & Age & Height $(\mathrm{cm})$ & Weight $(\mathrm{kg})$ \\
\hline Test group & $22.74 \pm 1.46$ & $182.42 \pm 2.97$ & $76.26 \pm 7.52$ \\
Control group & $23.68 \pm 1.77$ & $172.25 \pm 6.78$ & $78.23 \pm 5.23$ \\
$P$-value & 0.365 & 0.896 & 0.875 \\
\hline
\end{tabular}

TABLE 2: Statistics of the first four physical fitness indicators of the experimental group and the control group.

\begin{tabular}{lccc}
\hline Index & Test group & Control group & $P$-value \\
\hline $30 \mathrm{~m}$ sprint $(\mathrm{s})$ & $3.106 \pm 0.256$ & $3.256 \pm 0.026$ & 0.523 \\
$\begin{array}{l}\text { Squat jump height } \\
(\mathrm{cm})\end{array}$ & $62.35 \pm 6.23$ & $62.35 \pm 3.86$ & 0.309 \\
$\begin{array}{l}\text { Anaerobic peak power } \\
(\mathrm{w})\end{array}$ & $923.56 \pm 116.25$ & $902.36 \pm 96.42$ & 0.268 \\
Half squat 1RM (kg) & $105.36 \pm 6.38$ & $156.48 \pm 7.59$ & 0.879 \\
\hline
\end{tabular}

content was the same for 12 weeks, and each training session lasted $120 \mathrm{~min}$. The methods used in the control group are relatively simplified and involve few steps, while the methods used in the experimental group are more diversified and detailed.

\subsection{Experimental Content}

3.3.1. Training Content of the Experimental Group. The basic stage of core strength training is mainly to do some static movements on the sponge pad to improve the strength quality of the small waist and abdomen muscles, initially develop the ability of the neuro-muscular system, and lay the foundation for the later core strength training. The questionnaire survey by experts shows that the training methods and scores suitable for this stage are shown in Table 3:

Training plan: each training session adopts the cyclic practice method. Six training stations are set up in sequence. Six stations are completed continuously as a group; each station has an interval of $4 \mathrm{~min}$; and each group has an interval of $4 \mathrm{~min}$. The three groups of exercises A, B, and C are static exercises [24], and the average persistence time per test is $40.5 \mathrm{~s}$, so each training session takes $55 \mathrm{~s}$ for each group of exercises; the two groups $D$ and $E$ are dynamic exercises; each group exercises 15 times, e component left and right leg exercises, 5 times on each side, continuous completion. Experts" training situation for the consolidation phase of core strength training is shown in Table 4:

The core strength training improvement phase is based on the consolidation phase and gradually increases the difficulty of training, that is, reducing the support base, gradually increasing instability, gradually increasing the number of repetitions of dynamic movements, from one-dimensional movements to multi-dimensional movements, and so on. The training plan still adopts the cyclic practice method. Six training stations are set up in sequence [25]; six consecutive stations are formed as a group; the load of each group is 5 times; each 
TABle 3: Experts' scores on training methods at the basic stage of core strength training.

\begin{tabular}{lc}
\hline Training content & $\begin{array}{c}\text { Average } \\
\text { score }\end{array}$ \\
\hline Recumbent legs with arms stretched out and hips & 3.25 \\
straight & 2.5 \\
Supine arm support hip lift leg lift & 5 \\
Side-up arms, hips, and legs & 3.8 \\
Side-lying banana pose from both ends & 6 \\
Lie on your back with one knee and hip & \\
\hline
\end{tabular}

station has an interval of $5 \mathrm{~min}$; and each group has an interval of $6 \mathrm{~min}$.

3.3.2. Training Content of the Control Group. The control group used the traditional waist and abdomen strength training method and the circuit training method [26]; each exercise was done in six groups; the training time and the number of training for each group are 15 reps and 25 seconds, such as supine and prone movements; the coach makes each group do 25 reps or 25 seconds according to the athlete's state. The training situation is shown in Table 5:

\section{Experimental Analysis}

4.1. Analysis of Experimental Results. After 10 weeks of training, the performance of the experimental group was greatly improved compared with the control group. The 1RM modification rate of the experimental group was higher than that of the control group, but there was no significant difference. After 8 weeks of training, the vibration training group recorded a significant increase in the $1 \mathrm{RM}$ of the halfknee joint and a significant improvement. The increase in knee flexion of the experimental group was greater than that of the control group, but there was no significant difference. The increase in peak anaerobic strength of the experimental group was greater than that of the control group, but there was no significant difference. The comparison of 50-meter sprint, half-knee maximum strength, knee height, and peak anaerobic strength are shown in Figures 4 and 5.

\subsection{Comparative Analysis of Peak Moments of Hip and Knee} Joints. The maximum work represents the maximum onetime work value when a muscle group is repeatedly contracted for a certain number of times. It represents the functional strength of the muscle and reflects the maximum muscle strength to a certain extent. After the experiment, under the test conditions of $50 \%$ and $200 \%$ s, the extensor muscles of the experimental group increased by $56.5 \mathrm{~J}$ and $51.55 \mathrm{~J}$, respectively, and the increase rates were $24.72 \%$ and $19.66 \%$, respectively; the flexors increased by $20.36 \mathrm{~J}$ and $15 \mathrm{~J}$, respectively, and the increase rates were $19.36 \%$ and $30.2 \%$, respectively; there were significant differences in the growth of extensor and flexor muscles among the groups. In the control group, the extensor muscles increased by $85 \mathrm{~J}$ and $52 \mathrm{~J}$ under the test conditions of $60 \% \mathrm{~s}$ and $300 \%$, respectively, with an increase rate of $38.2 \%$ and $51.2 \%$, respectively; the flexors increased by $32.6 \mathrm{~J}$ and $22 \mathrm{~J}$, respectively, with an
TABLE 4: Experts' ratings of training methods in the consolidation phase of core strength training.

\begin{tabular}{lc}
\hline Training content & Average score \\
\hline Pull-in & 4.5 \\
Hip extension and knee flexion & 3.25 \\
Single-leg hip lift & 4.2 \\
Reverse back extension & 4.5 \\
\hline
\end{tabular}

increase rate of $36.25 \%$ and $37.28 \%$, respectively. The growth of extensor and flexor muscles has a very significant difference within the group $(P<0.01)$; comparing the groups, the growth of the extensor muscles has a significant difference, while the flexor muscles have no significant difference, as shown in Figure 6:

After the experiment, the relative peak torque of the knee joint flexors and extensors of the control and the experimental groups increased to varying degrees. The knee joints of the experimental group were at $50 \%, 150 \%$, and $200 \%$ s. Under the test conditions, the extensors increased by $1.32 \mathrm{Nm} / \mathrm{kg}, 1.35 \mathrm{Nm} / \mathrm{kg}$, and $1.26 \mathrm{Nm} / \mathrm{kg}$, respectively, and the increase rate was $11.25 \%, 12.36 \%$, and $14.36 \%$, respectively, while the knee flexors increased by $1.25 \mathrm{Nm} / \mathrm{kg}$, $1.17 \mathrm{Nm} / \mathrm{kg}$, and $1.19 \mathrm{Nm} / \mathrm{kg}$, respectively, and the increase rate was $20.3 \%, 23.56 \%$, and $22.6 \%$, respectively. Comparing the same group before and after the experiment, there was no significant difference in the growth of the extensor and the flexor muscle groups between two groups. Under the test conditions of $100 \% \mathrm{~s}, 150 \%$, and $200 \%$, the extensors increased by $1.2 \mathrm{Nm} / \mathrm{kg}, 1.5 \mathrm{Nm} / \mathrm{kg}$, and $1.3 \mathrm{Nm} / \mathrm{kg}$, respectively, with an increase rate of $25.6 \%, 25.36 \%$, and $27.3 \%$, respectively. Muscle increased by $1.3 \mathrm{Nm} / \mathrm{kg}, 1.56 \mathrm{Nm} / \mathrm{kg}$, and $1.2 \mathrm{Nm} / \mathrm{kg}$, respectively, and the increase was $15.36 \%$, $20.7 \%$, and $20.14 \%$, respectively. Comparing the same group before and after the experiment, there is a significant difference in the growth of the extensor muscle group at $50 \%$ and $200 \%$, while the growth of the flexor muscle group at $150 \%$ and $200 \%$ s has a significant difference. Comparing between groups, there is a significant difference in the growth of the extensor muscle group at $200 \%$, while the growth of the flexor muscle group at $150 \%$ s has a significant difference, as shown in Figure 7:

4.3. Analysis of Related Technologies. The programming of the number of sensors optimization scheme is mainly based on the local minimum principle to select the scheme. Therefore, the selection of the optimization scheme needs to be determined in conjunction with the maximum nondiagonal element change map of the MAC matrix. For the EI and MAC hybrid algorithm and the EIbased stepwise subtraction method, since the algorithm itself has a reduced number of measurement points, there may be only one local minimum. Under the condition of selecting three sensors, the hybrid algorithm may have only one or two minimum points. To facilitate comparison, the EI method uses the number of sensors determined by the stepwise accumulation method based on EI as a reference, and the hybrid algorithm of EI and MAC 
TABLE 5: Training content of lumbar and abdominal muscle strength training in the control group.

\begin{tabular}{lccc}
\hline Training content & Number of training groups & Training times (times) & Training time (s) \\
\hline Sit-ups & 6 & 25 & 20 \\
Hanging leg lift & 6 & 25 & 25 \\
Lie on your back & 6 & 25 & 25 \\
Prone from both ends & 6 & 25 & 25 \\
Weight-bearing body flexion and extension & 6 & 20 & 20 \\
Prone "back leg" & 6 & 25 & 20 \\
\hline
\end{tabular}

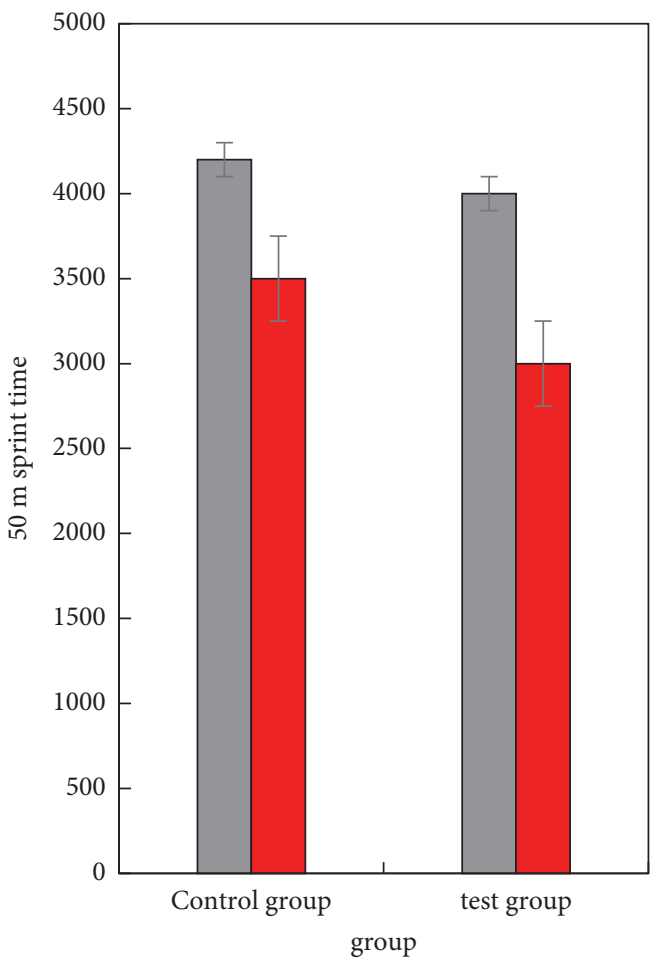

Before the experiment

After the experiment

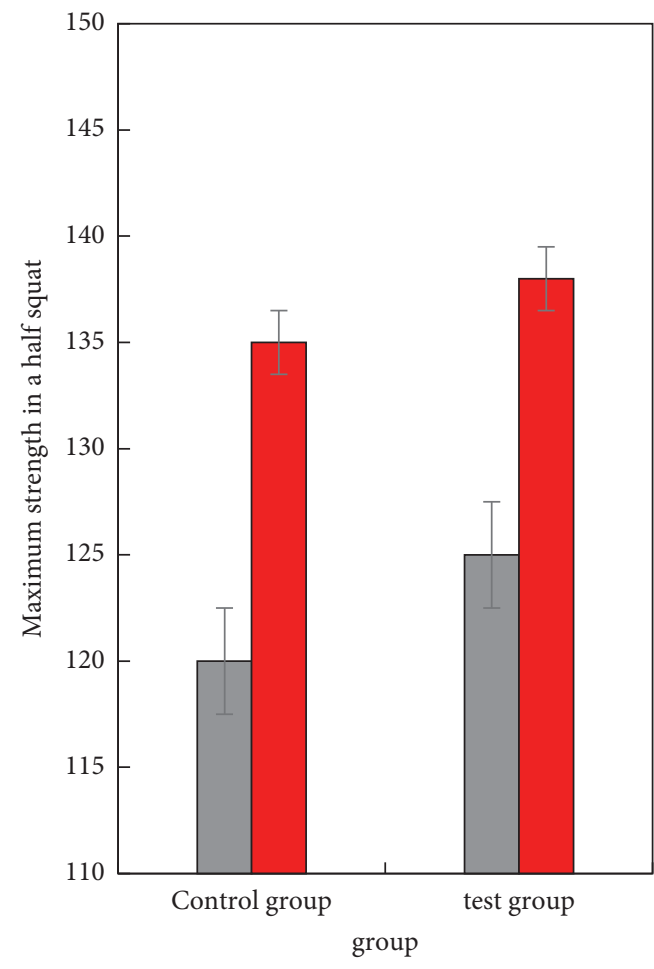

Before the experiment

After the experiment

Figure 4: $50 \mathrm{~m}$ Sprint and half squat maximum strength comparison.

uses the number of sensors determined by the stepwise subtraction method as a reference. To facilitate the observation of the six sensor optimization solutions corresponding to the maximum the changing law of nondiagonal elements, the data are converted into a graph, as shown in Figure 8.

It can be seen from Figure 8 that under the condition of six sensors, the EI-based stepwise subtraction method proposed in this paper can achieve the best measurement point layout effect. With the increase in the number of sensors, the measuring point arrangement scheme calculated by the gradual subtraction method presents a greater advantage. The measuring point scheme provided by the EI method also reflects the competitiveness in the case of multiple sensors. The gradual accumulation based on EI proposed in this paper law is also competitive. At the same time, it also shows that the number of sensors is not better, and a reasonable number of sensors need to be determined comprehensively according to the actual needs.
The measurement points selected by the effective independent method achieve maximum linear independence, including orthogonality, and the selection range is greater than orthogonality. In the case of fewer sensor measurement points, the measurement points selected by the effective independent method may not be able to take care of the installation of the sensor. When the added measurement points are better than orthogonal 45 in terms of linearity, the phenomenon shown in Figure 9 will appear. The 16, 17, 145, and 146 measuring points selected by the effective independent method in this example have good linear independence, which can ensure that the vertical 1st and $2 \mathrm{nd}, 1 \mathrm{st}$ and 4 th, 3rd, and 4 th orders have good positive values. However, the intersectionality cannot satisfy the orthogonality of the $2 \mathrm{nd}$ and $43 \mathrm{rd}$, 3rd, and 6th orders, and the eight measuring points are adjacent to each other, resulting in a decrease in the degree of visualization of the measured mode shape. Therefore, in the case of fewer sensor installation points, it is necessary to comprehensively consider the 


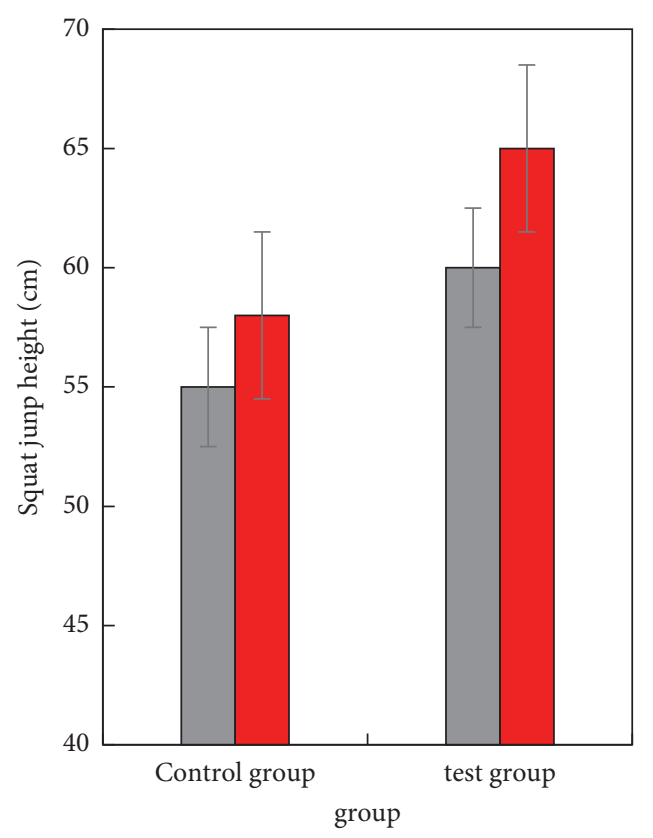

Before the experiment

After the experiment

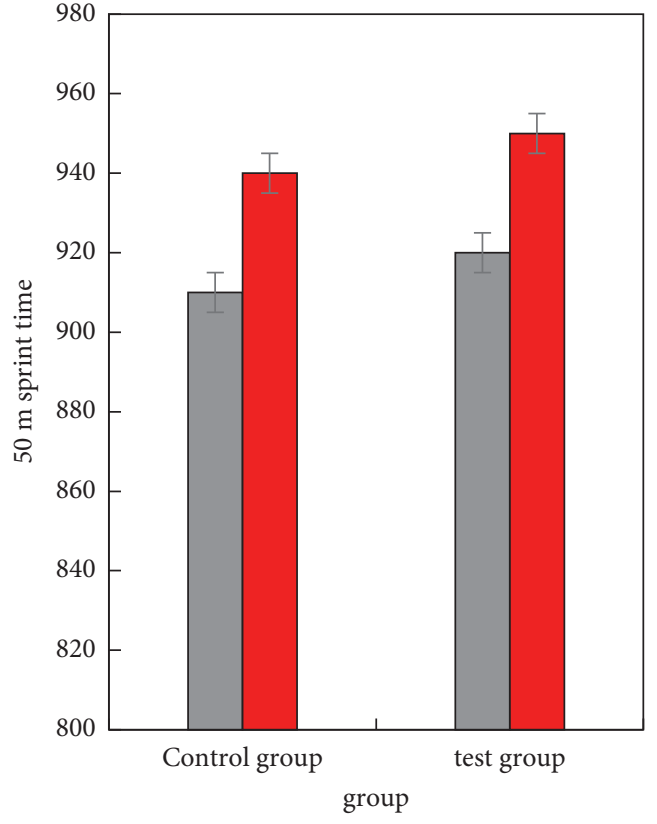

Before the experiment

After the experiment

FIgURE 5: Comparison of squat jump height and anaerobic peak power.
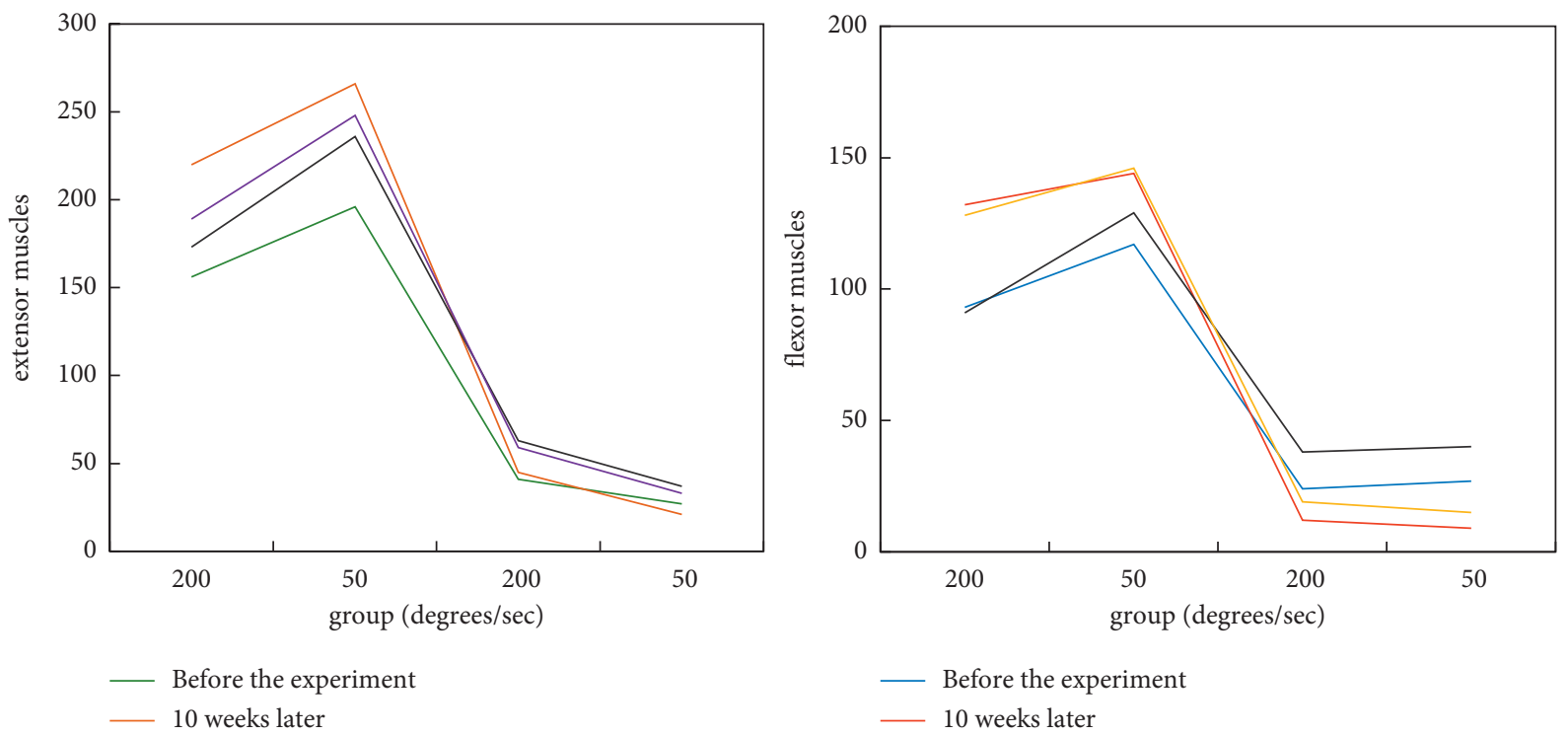

FIgURE 6: Diagram of changes in the maximum work of the hip joint.

optimal placement of sensors through a variety of optimization algorithms. The iteration times and maximum nondiagonal element changes of the two optimization algorithms are shown in Figures 9 and 10.

It can be seen from the figure that the effective independent method and the MAC hybrid algorithm have a relatively large amount of calculation, and the number of iterations is the same as the number of measuring points. Except for the effective independent method, several other optimization algorithms converge quickly during the initial iteration process and can the local minimum be reached at five sensors. 

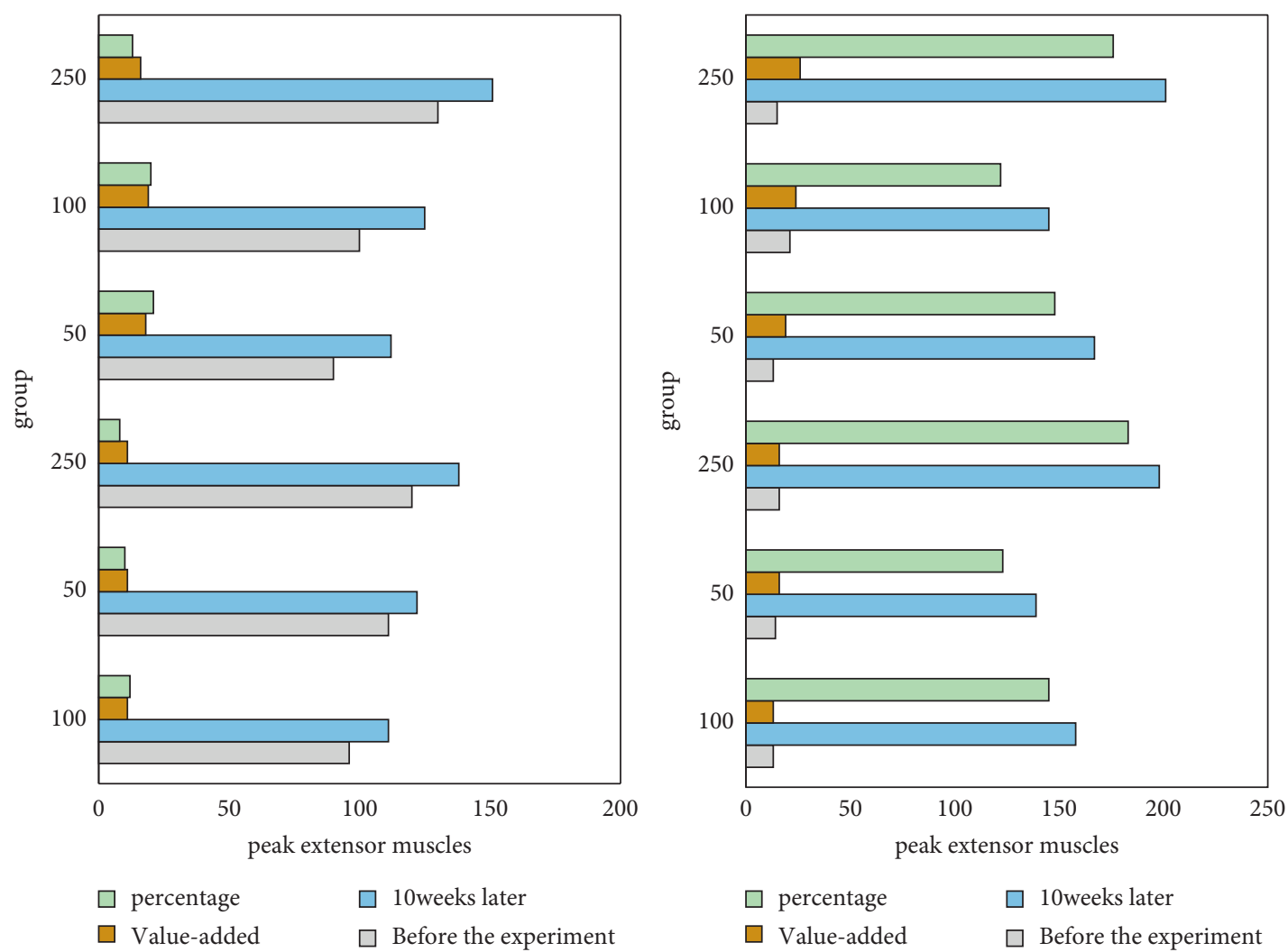

$\square$ percentage

$\square$ 10weeks later

$\square$ Value-added

$\square$ Before the experiment

$\square$ Value-added

$\square$ Before the experiment

FIgURE 7: Changes in peak torque of the knee joint group.



FIgURE 8: The sensor optimization scheme corresponds to the change of the largest nondiagonal element. 


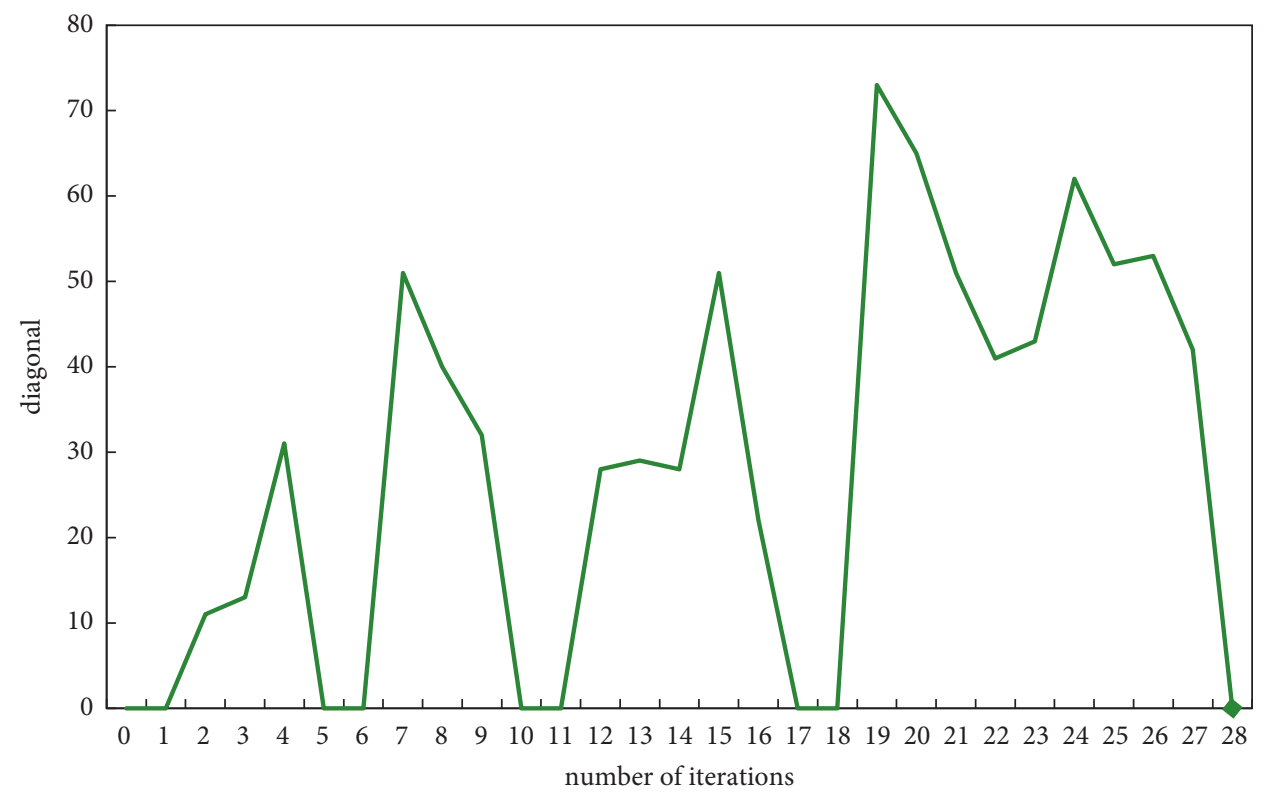

$\longrightarrow$ EI method diagonal element change diagram

FIgURE 9: Diagonal element change diagram of EI method.



FIgUre 10: The maximum nondiagonal element change diagram of the EI and MAC hybrid algorithm.

\section{Conclusions}

In this paper, the advantages and disadvantages of existing motion sensors are analyzed, and it has been finally decided to select six-axis three-degree-of-freedom sensing MPU6050 to detect the object motion state and select "amplitudelimited filtering algorithm + complementary filtering algorithm" to optimize and integrate the motion data, to improve the accuracy of motion state analysis. The main research work in this paper is as follows: the "+ complementary filter" algorithm is used to optimize the data and complete the software design; the motion state data are read with the upper machine; and the actual motion state information are compared. The sports performance of the world's outstanding men's 110-meter hurdles must be continuously improved. China's hurdles have made breakthrough progress in recent years, but there is still a certain gap compared with foreign outstanding athletes. The average sports performance of Chinese athletes is much lower than that of high-level athletes in the world. This directly reflects the lack of physical training of Chinese athletes. In particular, Chinese athletes are much worse than 
foreign athletes in terms of speed quality. This is also a warning. We must continue to study and discover the characteristics of athletes, conduct scientific and systematic analysis, and further improve the athlete's speed quality. The experimental results showed that the extensor muscles in the experimental group increased by $56.5 \mathrm{j}$ and $51.55 \mathrm{j}$, respectively, with an increase rate of $24.72 \%$ and $19.66 \%$, respectively. The extensor muscle of the control group increased by $85 j$ and $52 j$ under the test conditions of $60 \%$ and $300 \%$, respectively, with the increase rates of $38.2 \%$ and $51.2 \%$, respectively; Flexor muscles increased by $32.6 \mathrm{j}$ and $22 \mathrm{j}$, respectively, with an increase rate of $36.25 \%$ and $37.28 \%$, respectively.

\section{Data Availability}

Data sharing is not applicable to this article as no data sets were generated or analyzed during the current study.

\section{Conflicts of Interest}

The authors declare that there are no conflicts of interest regarding the publication of this article.

\section{References}

[1] R. Parada, J. Melià-Seguí, and R. Pous, "Anomaly detection using rfid-based information management in an IOT context," Journal of Organizational and End User Computing, vol. 30, no. 3, pp. 1-23, 2018.

[2] A. M. Pinto, A. P. Moreira, and P. G. Costa, "WirelessSyncroVision: wireless synchronization for industrial stereoscopic systems," International Journal of Advanced Manufacturing Technology, vol. 82, no. 5-8, pp. 909-919, 2016.

[3] M. E. Holmstrup, B. T. Jensen, W. S. Evans, and E. C. Marshall, "Eight weeks of kettlebell swing training does not improve sprint performance in recreationally active females," International Journal of Exercise Science, vol. 9, no. 3, pp. 437-444, 2016.

[4] N. Dobbin, J. Highton, S. L. Moss, and C. Twist, "The effects of in-season, low-volume sprint interval training with and without sport-specific actions on the physical characteristics of elite academy rugby league players," International Journal of Sports Physiology and Performance, vol. 15, no. 5, pp. 705-713, 2019.

[5] H.-J. Gil and J.-H. Lee, "A study on the characteristics of $110 \mathrm{~m}$ hurdle 7-step strategy: a pilot study," Korean Journal of Sports Science, vol. 26, no. 3, pp. 1311-1320, 2017.

[6] W. Misaki, S. Yasuo, R. Nagahara, A. Matsuo, and A. Nagano, "Step-to-step analysis of anteroposterior ground reaction force during $110 \mathrm{M}$ hurdle," ISBS Proceedings Archive, vol. 36, no. 1, p. 90, 2018.

[7] A. Camacho-Cardenosa, M. Camacho-Cardenosa, I. Martínez-Guardado, J. Brazo-Sayavera, R. Timon, and G. Olcina, "Effects of repeated-sprint training in hypoxia on physical performance of team sports players," Revista Brasileira de Medicina do Esporte, vol. 26, no. 2, pp. 153-157, 2020.

[8] S. N. Mohanty, E. L. Lydia, M. Elhoseny, M. Majid, G. Al Otaibi, and K. Shankar, "Deep learning with LSTM based distributed data mining model for energy efficient wireless sensor networks," Physical Communication, vol. 40, no. 3, https://doi.org/10.1016/j.phycom.2020.101097 In press, Article ID 101097, 2020.

[9] H. J. Lim, Y. J. Kang, and J. Y. Song, "Development of a mobile game and wearable device for upper limb rehabilitation after brain injury," Journal of Rehabilitation Welfare Engineering \& Assistive Technology, vol. 11, no. 3, pp. 253-259, 2017.

[10] D. Wu, D. Chatzigeorgiou, K. Youcef-Toumi, and R. BenMansour, "Node localization in robotic sensor networks for pipeline inspection," IEEE transactions on industrial informatics, vol. 12, no. 2, pp. 809-819, 2016.

[11] A. Philipose and A. Rajesh, "Investigation on energy efficient sensor node placement in railway systems," Engineering Science and Technology, an International Journal, vol. 19, no. 2, pp. 754-768, 2016.

[12] D. Wu, D. Chatzigeorgiou, K. Youcef-Toumi, and R. BenMansour, "Node localization in robotic sensor networks for pipeline inspection," IEEE Transactions on Industrial Informatics, vol. 12, no. 2, p. 1, 2016.

[13] S. Mukherjee, S. Nandy, and A. Dey, "Development of wildlife conservation system using energy-efficient real-time sensor networking," International Journal of Engineering and Advanced Technology, vol. 9, no. 4, pp. 866-874, 2020.

[14] H.-W. Lee, J.-C. Chun, and W.-G. Jeong, "Removing the motion artifacts in the pulse signal detected from the PFS using the Quasi-periodicity," Journal of Korea Institute of Information, Electronics, and Communication Technology, vol. 9, no. 6, pp. 591-598, 2016.

[15] S. H. Hwang, K. R. Ko, and S. B. Pan, "Golf swing analysis system using camera and inertial sensor," The Journal of Korean Institute of Information Technology, vol. 15, no. 4, pp. 139-147, 2017.

[16] H. M. Do, H.-S. Kim, D. I. Park et al., "Intuitive teaching device mountable on a robotic arm for efficient collaboration between humans and robots," Journal of The Korean Society of Manufacturing Technology Engineers, vol. 26, no. 6, pp. 577583, 2017.

[17] N. Kim, "A study on the implementation of transmission type PPG measurement device in a wrist watch," Journal of Korea Institute of Information, Electronics, and Communication Technology, vol. 10, no. 2, pp. 161-167, 2017.

[18] C.-S. Kim and S.-H. Jung, "A MEMS-based finger wearable computer input devices," Journal of the Korea Institute of Information and Communication Engineering, vol. 20, no. 6, pp. 1103-1108, 2016.

[19] Z. Y. Shi, "Application of motion capture technology based on MEMS sensor in sports training," Revista de la Facultad de Ingenieria, vol. 32, no. 14, pp. 303-308, 2017.

[20] L. Lamprecht, R. Ehrenpfordt, T. Zoller, and A. Zimmermann, "Application of human motion energy harvesters on industrial linear technology," IET Wireless Sensor Systems, vol. 9, no. 2, pp. 53-60, 2019.

[21] J. Hanca, G. Braeckman, A. Munteanu, and W. Philips, "Lightweight real-time error-resilient encoding of visual sensor data," Journal of Real-Time Image Processing, vol. 12, no. 4, pp. 1-15, 2014.

[22] M. Haghi, K. Thurow, and R. Stoll, "A ubiquitous and configurable wrist-worn sensor node in hazardous gases detection," Advances in Science, Technology and Engineering Systems Journal, vol. 3, no. 5, pp. 248-257, 2018.

[23] I. Khoufi, P. Minet, A. Laouiti, and S. Mahfoudh, "Survey of deployment algorithms in wireless sensor networks: coverage and connectivity issues and challenges," International Journal of Autonomous and Adaptive Communications Systems, vol. 10, no. 4, pp. 341-390, 2017. 
[24] M. A. A. Mamun, M. A. Hannan, A. Hussain, and H. Basri, "Theoretical model and implementation of a real time intelligent bin status monitoring system using rule based decision algorithms," Expert Systems with Applications, vol. 48, pp. 76-88, 2016.

[25] X. Liu, M. Zhang, and V. Jan, "A low-power multifunctional CMOS sensor node for an electronic façade," IEEE Transactions on Circuits \& Systems I Regular Papers, vol. 61, no. 9, pp. 2550-2559, 2017.

[26] Y. Li and Y. Yang, "The research and implementation of dynamic loader for sensor network nodes," Chinese Journal of Sensors and Actuators, vol. 31, no. 7, pp. 1113-1117, 2018. 